\title{
Quantitative Equidistribution for Certain Quadruples in Quasi-Random Groups: Erratum
}

\author{
TIM AUSTIN ${ }^{\dagger}$ \\ Courant Institute, New York University, New York, NY 10012, USA \\ (e-mail: tim@cims.nyu.edu)
}

In my recent paper [1] there is a mistake in the proof of Corollary 3 . The first line of the displayed equation in that proof asserts that

$$
\int_{G}\left|\left\langle u, \pi^{g} v\right\rangle_{V}\right|^{2} \mathrm{~d} g=\int_{G}\left\langle u \otimes u,\left(\pi^{g} \otimes \pi^{g}\right)(v \otimes v)\right\rangle_{V \otimes V} \mathrm{~d} g .
$$

However, since the paper uses complex-valued representations, the integrand on the right here may not retain the absolute value of that on the left. Without this equality, the proof of Corollary 3 can no longer be reduced to an application of Lemma 2. However, it can be proved directly from Schur Orthogonality along very similar lines to the proof of Lemma 2.

Indeed, replacing $u$ with $u-P_{\pi}^{\circ} u$ and $v$ with $v-P_{\pi}^{\circ} v$, we may assume that $\pi$ has no trivial component. Let $\pi=\bigoplus_{i \geqslant 1} \rho_{i}, u=\bigoplus_{i \geqslant 1} u_{i}$ and $v=\bigoplus_{i \geqslant 1} v_{i}$ be a decomposition into irreducible components as in the original proof of Lemma 2. Substituting into the desired integral gives

$$
\int_{G}\left|\left\langle u, \pi^{g} v\right\rangle_{V}\right|^{2} \mathrm{~d} g=\int_{G}\left\langle u, \pi^{g} v\right\rangle \overline{\left\langle u, \pi^{g} v\right\rangle} \mathrm{d} g=\sum_{i, j \geqslant 1} \int_{G}\left\langle u_{i}, \rho_{i}^{g} v_{i}\right\rangle_{V_{i}} \overline{\left\langle u_{j}, \rho_{j}^{g} v_{j}\right\rangle_{V_{j}}} \mathrm{~d} g .
$$

A standard version of Schur Orthogonality (see, for instance, [2, Theorems 2.3 and 2.4]) evaluates each term in this sum to give that it equals

$$
\sum_{\substack{i, j \geqslant 1 \\ \rho_{i} \cong \rho_{j}}} \frac{1}{\operatorname{dim}\left(V_{i}\right)}\left\langle u_{i}, u_{j}\right\rangle_{V_{i}}\left\langle v_{i}, v_{j}\right\rangle_{V_{i}}
$$

Here we have used that when $\rho_{i} \cong \rho_{j}$, the isomorphism is unique, and so we may canonically identify $u_{j}$ and $v_{j}$ with elements of $V_{i}$ to compute the inner products.

$\dagger$ Research supported by a fellowship from the Clay Mathematics Institute. 
Finally, since $G$ is $D$-quasi-random and $\pi$ contains no trivial subrepresentation, the above sum is bounded by

$$
\begin{aligned}
& D^{-1} \sum_{\substack{i, j \geqslant 1 \\
\rho_{i} \cong \rho_{j}}}\left\|u_{i}\right\|_{V_{i}} \cdot\left\|u_{j}\right\|_{V_{j}} \cdot\left\|v_{i}\right\|_{V_{i}} \cdot\left\|v_{j}\right\|_{V_{j}} \leqslant D^{-1}\left(\sum_{i \geqslant 1}\left\|u_{i}\right\|_{V_{i}}\left\|v_{i}\right\|_{V_{i}}\right)^{2} \\
& \leqslant D^{-1}\left(\sum_{i \geqslant 1}\left\|u_{i}\right\|_{V_{i}}^{2}\right)\left(\sum_{i \geqslant 1}\left\|v_{i}\right\|_{V_{i}}^{2}\right)=D^{-1}\|u\|_{V}^{2}\|v\|_{V}^{2}
\end{aligned}
$$

where the second estimate is by the Cauchy-Bunyakowski-Schwarz inequality. Thus, we have actually obtained a strengthening of the original Corollary 3 with $D^{-1 / 2}$ replaced by $D^{-1}$.

\section{References}

[1] Austin, T. (2015) Quantitative equidistribution for certain quadruples in quasi-random groups. Combin. Probab. Comput. 24 376-381.

[2] Bump, D. (2004) Lie Groups, Vol. 225 of Graduate Texts in Mathematics, Springer. 